\title{
Surfactant films in lyotropic lamellar (and related) phases: Fluctuations and interactions
}

\author{
Frédéric Nallet \\ Université de Bordeaux. Centre de recherche Paul-Pascal-CNRS \\ 115 avenue du Docteur-Schweitzer \\ F-33600 Pessac, FRANCE
}

This paper is dedicated to Dr Dominique LANGEvin on the occasion of her $70^{\text {th }}$

birthday

\begin{abstract}
The analogy between soap films thinning under border capillary suction and lamellar stacks of surfactant bilayers dehydrated by osmotic stress is explored, in particular in the highly dehydrated limit where the soap film becomes a Newton black film. The nature of short-range repulsive interactions between surfactant-covered interfaces and acting across water channels in both cases will be discussed.
\end{abstract}

\section{Introduction}

Bubble coalescence in foams obviously involves the rupture of the liquid film separating adjacent bubbles. However, this phenomenon still remains not well understood in the presence of surface-active agents [1]. A freely-suspended soap film is a convenient model for studying liquid films and, when drawn horizontally, the film thins as long as capillary forces are not opposed by the repulsive component of interactions across the water channel. The interactions between the two surfactant-covered air-water interfaces (with both repulsive and attractive components in general) give rise to the so-called disjoining pressure [2,3]. If the film does not rupture before reaching a force cancellation point, two states are possible, differing by the thickness $h$ of their water channels. They are commonly described as "common black films" (CBF) or "Newton black films" (NBF) for the thicker or the thinner states, respectively. Following the analysis proposed by VRIJ [4, the common black film may actually not be a stable state because spontaneous (thermally-excited) thickness fluctuations around the film average thickness $h_{\mathrm{CBF}}$-fluctuations also pictorially described as "peristaltic fluctuations" [5]-become unstable under particular circumstances when their wavelength is larger than a critical value $\lambda_{c}$. For the instability to occur, the repulsive component of the interaction (often considered to be of electrostatic 
origin) should combine with van der Waals attractions to yield an interaction potential $V(h)$ as a function of $h$ exhibiting a concave shape-see Fig. 1. In other

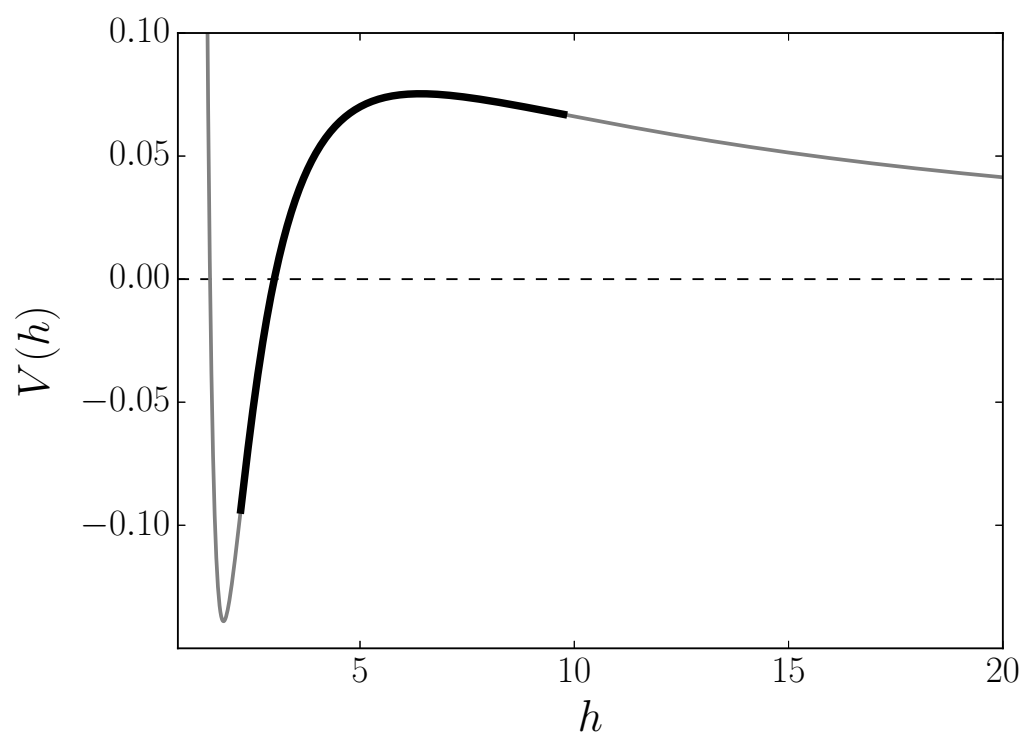

Figure 1: Interaction potential $V$ vs. separation $h$ curve exhibiting a region where $\partial^{2} V / \partial h^{2}<0$, which leads to a peristaltic instability. Newton (respectively common) black films correspond to the light-grey region of the curve on the left (resp. right) of the thick black region

words $\partial^{2} V / \partial h^{2}<0$ when evaluated at $h=h_{\mathrm{CBF}}$ leads, generally speaking, to an unstable common black film.

In Ref. 4, the critical wavelength is given by:

$$
\lambda_{c}=\left[-2 \pi^{2} \gamma \times \frac{1}{\left.\frac{\partial^{2} V}{\partial h^{2}}\right|_{h_{\mathrm{CBF}}}}\right]^{1 / 2}
$$

with $\gamma$ the interfacial tension. This implies that films of finite size $L$ remain robust against peristaltic destabilisation as long as $\lambda_{c}$ remains larger than $L$.

In cases where a common black film of thickness $h=h_{\mathrm{CBF}}$ is unstable, and within the framework of VRIJ's analysis, diverging peristaltic fluctuations lead to a Newton black film only if $V$ changes again its concavity at some thickness $h<h_{\mathrm{CBF}}$. This requires the presence of short range repulsive forces between the two surfactant-covered air-water interfaces, or a physical mechanism generating effective repulsive forces strong enough to overcome van der Waals attractions. This is indeed the case for the toy model leading to the curve $V(h)$ displayed in Fig. 1, but a clear physical model for such a strong repulsion is still lacking. 
Experimentally, it is observed that soap films drawn from too dilute surfactant solutions do not form Newton black films (see, for instance, Ref. [6]), but rupture instead, the (qualitative) explanation being that the two facing surfactant layers are then not dense enough for generating the required repulsion [1, 6]. Conversely, highly-loaded surfactant layers should then always lead to stable Newton black films, following the same reasoning. If correct, the instabilitydriven CBF-to-NBF transition would then be quite similar to spinodal decomposition [7, with common and Newton black films being analogous to the gas and liquid phases, respectively, $\partial^{2} V / \partial h^{2}$ being somehow analogous to the bulk compressibility in the phase separation analogue. The (dilute) gas phase "collapses" into a denser liquid phase owing to long-range, attractive interactions but strong, short-range repulsions prevent a further "big crunch" at infinite densities. As is well known, these short-range repulsions between the molecular components of the liquid or gas phases are actually effective interactions, commonly explained as arising from Pauli repulsion of overlapping electron orbitals [8]. There is unfortunately no such widely accepted explanation for the mechanism stabilising Newton black films, authors often referring for instance to "subtle and complex forces associated with the structure of water" [9], to "short-range entropic confinement forces" [10] or to "non-conventional interactions of entropic origin" [11].

The rupture of the Newton black film is beyond the spinodal decomposition analogy, of course, and cannot be addressed within the framework of VRIJ's analysis: As already mentioned, there is no clear mechanism implementing another curvature inversion in the disjoining pressure equation of state for channel thicknesses smaller than $h_{\mathrm{NBF}}$. A key ingredient could be taking into account surface elasticity, because it controls the amplitude of thermal fluctuations in the surfactant layer density [1,5, 10, 12, 14]. What is still lacking, however, is a mechanism coupling then explicitly surface elasticity-an "in-plane" propertyto disjoining pressure, acting in the direction perpendicular to the surfactantcovered interfaces, even though at least one proposition has been formulated in the past [5].

Another analogy is also quite relevant in the understanding of soap films. The so-called lyotropic lamellar phases are prepared by mixing appropriate amounts of lipids, soaps or surfactants with a solvent-water, to be specific-in conditions where self-assembly leads to the formation of bilayers of surface-active molecules, separated by water channels and periodically stacked along an axis, $z$, perpendicular to their average plane [15. Such system are structurally close to soap films. By controlling the osmotic pressure $\Pi_{o}$ of water, as pioneered in Ref. [16, the thickness of the water channels can be varied and, in the limit of high pressures, strongly dehydrated lamellar phases are obtained. Travelling along $z$ for one stacking period $\ell$, one would go from half a bilayer to water to half a bilayer again and thus describe the classical three-layer structure of a soap film [17. When the thickness of the water core is comparable to, or even less than the bilayer thickness, there is little structural difference (not considering the gas phase, though) between a Newton black film and a lamellar phase at scale $\ell$. 
Three-component lamellar phases, with both oil and water swelling each the appropriate side of surfactant monolayers, would be even better analogues to Newton black films at simultaneously high oil and low water contents, a situation also encountered in adhesive oil-in-water emulsions [18, and rather close (upon interchange between oil and water phases) to the so-called "grey films" in water-in-oil emulsions [19]. Interactions between surfactant-covered oil-water interfaces, across the oil channel as well as across the water channel, have indeed been studied (with a different perspective, though) in such threecomponent lamellar systems [20], but not in the limiting case of a very thin water channel. Besides, quantitative studies of interactions in oil-in-water emulsions when oil droplets are close to molecular contact remain scarce [21,22].

\section{The two-component lyotropic lamellar phase}

From a thermodynamic point of view, the description of a two-component system requires a single composition variable, for instance the "solute" (surfactant, in the present context) volume fraction $\phi$, as it is reasonably safe to assume incompressibility. From a structural point of view, a minimal set of two variables should also be introduced for describing a lamellar stack of bilayers: the stacking period $\ell$ and a surface concentration, for instance $\Gamma$, the number of surfactant molecules per unit monolayer area. Within simple geometric assumptions, these three variables are not independent. Assuming homogeneous and ideally flat bilayers, a simple geometric description gives

$$
\ell \phi=2 v \Gamma
$$

with $v$ the volume of a surfactant molecule-a well-defined constant quantity owing to incompressibility. If, as an additional hypothesis, the surface concentration $\Gamma$ is constant, eq. (2) becomes the well-known one-dimensional "swelling law", experimentally satisfied in numerous (rather dilute) systems (one example, among many others, may be found in Ref. [23]), sometimes up to a small logarithmic correction attributed to the thermally-induced crumpling of the bilayers 24.

It has been observed long ago, however, that $\Gamma$ experiences significant variations in the opposite limit of dehydrated lamellar phases. Relevant examples, among many others, may be found in Ref. [25] 28]. In qualitative terms, this is easily understood: For a given composition $\phi$, the ratio $\ell / \Gamma$ is constant, and a compromise should be found between inter-bilayer interactions (acting along the stacking axis $z$ ), favouring large $\ell$ when repulsive, and intra-bilayer (in-plane) interactions, favouring an "optimal" interfacial coverage $\Gamma^{*}$, presumably close to parameter $\Gamma_{\infty}$ in Langmuir, Frumkin and other equations of state describing surfactant adsorption at the air-water interface-see, for instance, Ref. [29] for more details. Upon dehydration, as $z$-interactions become stronger, $\ell$ "wins" and the surface concentration is bound to increase above $\Gamma^{*}$. The model recently proposed in Ref. [30, recalled briefly below, similar in spirit to the model found 
in Ref. [31] but in a case where electrostatic interactions are irrelevant, gives an explicit physical mechanism for such a behaviour.

\subsection{Thermodynamic considerations}

The model starting point is the core part of the Gibbs free energy density, viz. the excess (Helmholtz) free energy density $f_{\text {exc }}$ as a function of two among the three relevant parameters $\ell, \phi$ and $\Gamma$, considering eq. (2) to be valid. Choosing composition variables $\phi$ and $\Gamma$ :

$$
f_{\text {exc }}(\phi, \Gamma)
$$

explicit forms for the "dilution law", or longitudinal equation of state $\ell(\phi) \equiv$ $2 v \Gamma_{\text {opt }}(\phi) / \phi$, as well as for the lateral equation of state $\Gamma_{\text {opt }}(\phi)$ are deduced from the minimisation equation

$$
\frac{\partial f_{\mathrm{exc}}}{\partial \Gamma}=0
$$

when an explicit form for $f_{\text {exc }}$ is chosen.

In Ref. [30, the free energy density is built by adding "longitudinal" and "lateral" contributions, the former accounting for the periodic stacking, and the latter for the stacked objects, viz. bilayers. Owing to their two-dimensional nature, bilayers are subjected to conformational fluctuations (or "undulations") with a specific character, as the mean square amplitude of undulation fluctuations for an isolated bilayer is divergent in the limit of infinite lateral extension. In a lamellar phase, bilayers are stacked and large amplitude undulations are prohibited to an extent that depends on the thickness of the water channels. In the analysis given by HELFRICH [32, the resulting configurational entropy penalty is estimated as

$$
f_{\text {und }}=\frac{1}{\ell} \times \frac{3 \pi^{2}}{128} \frac{\left(k_{B} T\right)^{2}}{\kappa} \frac{1}{(\ell-\delta)^{2}}
$$

(here written with a $-T$ factor to become a free energy density) in terms of bilayer bending modulus $\kappa$ and bilayer thickness $\delta$. Note that, similarly to the VAN DER WAALS "correction" to the ideal gas free energy accounting for excluded volume effects, with a divergence in the high density limit, eq. (5) diverges in the wholly dehydrated limit where $\ell \rightarrow \delta$ and that, when dealing with non-ideal gaseous phases, this feature has been criticised long ago [33].

In lamellar phases, interactions between bilayers, including van der Waals attractive forces of course, have to be taken into account, as already recognised in Ref. 32. But, as argued by MiLner and Roux [34, one should not merely add to the entropic term in the free energy the interaction potentials relevant for the forces being considered, but rather incorporate their effects as a virial term. This amounts to adding a term

$$
f_{\mathrm{vir}}=-k_{B} T \chi \phi^{2}
$$


where $\chi$ measures the average strength of the inter-bilayer interactions across the whole range of possible separations, similarly to the measure provided by the second virial coefficient for inter-molecular interactions in the classical theory of liquid-gas phase separation.

As far as the "lateral" contribution to the free energy is concerned, inspiring ideas may be found by considering the related problem of the equation of state of surface-adsorbing molecules (or particles) accounting for both excluded area and in-plane interactions, as discussed-for instance-in Ref. [29, 35] and [36], respectively. For the present purpose, the "van der Waals" approach has been chosen, with the corresponding free energy expression

$$
f_{2 \mathrm{D}}=\frac{k_{B} T}{\ell} \times\left\{\Gamma \ln \left(\frac{\Gamma}{\Gamma_{\infty}-\Gamma}\right)+\frac{1}{2} b_{2} \Gamma^{2}\right\}
$$

parameters $\Gamma_{\infty}$ and $b_{2}$ describing, respectively, the upper limit for surfactant adsorption (excluded area) and, in terms of a second virial coefficient, in-plane (cohesive) interactions.

With the above-described physical ingredients, the model for the free energy density becomes 30]:

$$
\begin{aligned}
f_{\mathrm{exc}}= & \frac{1}{\ell} \times \frac{3 \pi^{2}}{128} \frac{\left(k_{B} T\right)^{2}}{\kappa} \frac{1}{(\ell-\delta)^{2}} \\
& -k_{B} T \chi \phi^{2}+\frac{k_{B} T}{\ell} \times\left\{\Gamma \ln \left(\frac{\Gamma}{\Gamma_{\infty}-\Gamma}\right)+\frac{1}{2} b_{2} \Gamma^{2}\right\}
\end{aligned}
$$

which is also, introducing reduced quantities:

$$
\begin{aligned}
\tilde{f}_{\text {exc }}= & \frac{3 \pi^{2}}{128} \times \frac{1}{\tilde{\kappa}} \times \frac{\phi^{3}}{\tilde{\Gamma}^{3}\left(1-\phi \Gamma_{0} / \tilde{\Gamma}\right)^{2}} \\
& -\tilde{\chi} \phi^{2}+\frac{\phi}{2}\left\{\ln \left(\frac{\tilde{\Gamma}}{1-\tilde{\Gamma}}\right)+\frac{1}{2} b_{2} \tilde{\Gamma}\right\}
\end{aligned}
$$

with $\tilde{f}_{\mathrm{exc}} \equiv v f_{\mathrm{exc}} /\left(k_{B} T\right), \tilde{\Gamma} \equiv \Gamma / \Gamma_{\infty}, \tilde{\kappa} \equiv 8 v^{2} \Gamma_{\infty}^{3} \kappa /\left(k_{B} T\right), \Gamma_{0} \equiv \delta /\left(2 v \Gamma_{\infty}\right)$, $\tilde{\chi} \equiv \chi v$ and $\tilde{b}_{2} \equiv b_{2} \Gamma_{\infty}$.

Implicit in the four-parameter model given above, possible dependences of $\tilde{\kappa}$ on $\tilde{\Gamma}$, and of $\tilde{\chi}$ on $\phi$ may be introduced. On the other hand, even if nothing prevents in HELFRICH's analysis $\Gamma_{0} \neq 1$, it is presumably legitimate to assume $\Gamma_{0}=1$, thereby reducing the number of model parameters to three. The most simple case, viz. $\tilde{\kappa}$ and $\tilde{\chi}$ constant, as well as $\Gamma_{0}=1$, will now be discussed.

\subsubsection{In-plane elasticity}

A first direct consequence of the above-described model concerns the optimal surface coverage, solving eq. (4) for $\Gamma_{\text {opt }}$. Fig. 2 displays $f_{\text {exc }}$ as a function of $\Gamma$, eq. (9)-note that reduced units are from now on implicitly assumed-, for 


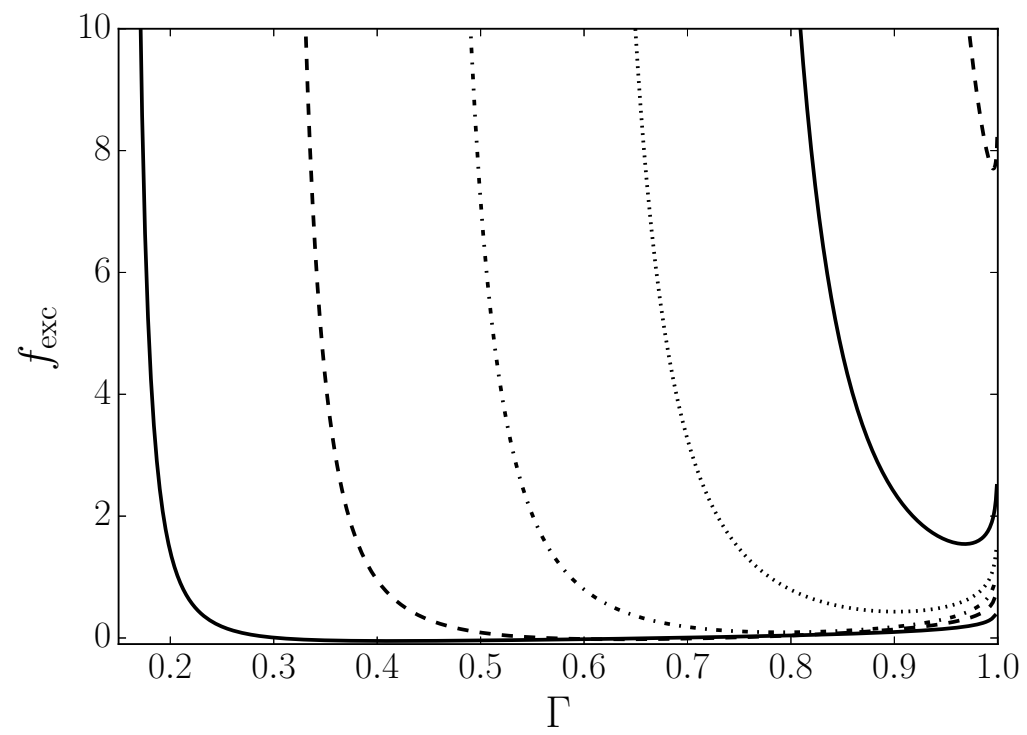

Figure 2: Model free energy density as a function of surface coverage-dilution effect. Surfactant volume fractions $\phi: 0.15$ (solid line), 0.29 (dashed line), 0.43 (dash-dotted line), 0.57 (dotted line), 0.71 (solid line) and 0.85 (dashed line). Model parameters: $\kappa=1$., $\chi=1.55, b_{2}=-1$.

representative values of the bilayer volume fractions and arbitrarily chosen, but fixed values of the model parameters $\kappa, \chi$ and $b_{2}$. The displacement towards higher optimal surface coverage upon dehydrating the lamellar stack evidences the " $\ell-\Gamma$ " interplay, eq. (2), with stronger interactions along the stacking axis $z$ more easily overcoming in-plane interactions.

As apparent in Fig. 2, dilution not only changes the optimal surface coverage $\Gamma_{\text {opt }}$, but also surface elasticity as an indirect consequence of the " $\ell-\Gamma$ " interplay. The $f_{\text {exc }}(\phi, \Gamma)$ curves are indeed much flatter in the vicinity of $\Gamma_{\text {opt }}$ at higher hydration. As made clear in Fig. 3. however, the most direct role-as far as surface elasticity is concerned-should be attributed to in-plane interactions, monitored by the cohesion parameter $b_{2}$. While the optimal surface coverage practically does not depend on bilayer cohesion $\left(\Gamma_{\text {opt }}\right.$ varies by less than $0.3 \%$ for the conditions chosen in Fig. (3), the free energy density curvature is significantly altered.

While the inter-bilayer interaction parameter $\chi$ has no effect on surface elasticity (from eq. (9), $\partial^{2} f_{\text {exc }} / \partial \Gamma^{2}$ does not depend on $\chi$, obviously), the bilayer bending modulus $\kappa$ plays some role in this respect, though less directly than $b_{2}$, and again through the " $\ell-\Gamma$ " interplay: A smaller value for $\kappa$ indeed implies a larger configurational entropy penalty, resulting in a stronger effective inter-bilayer repulsion. This leads in turn to a denser interfacial coverage and, 


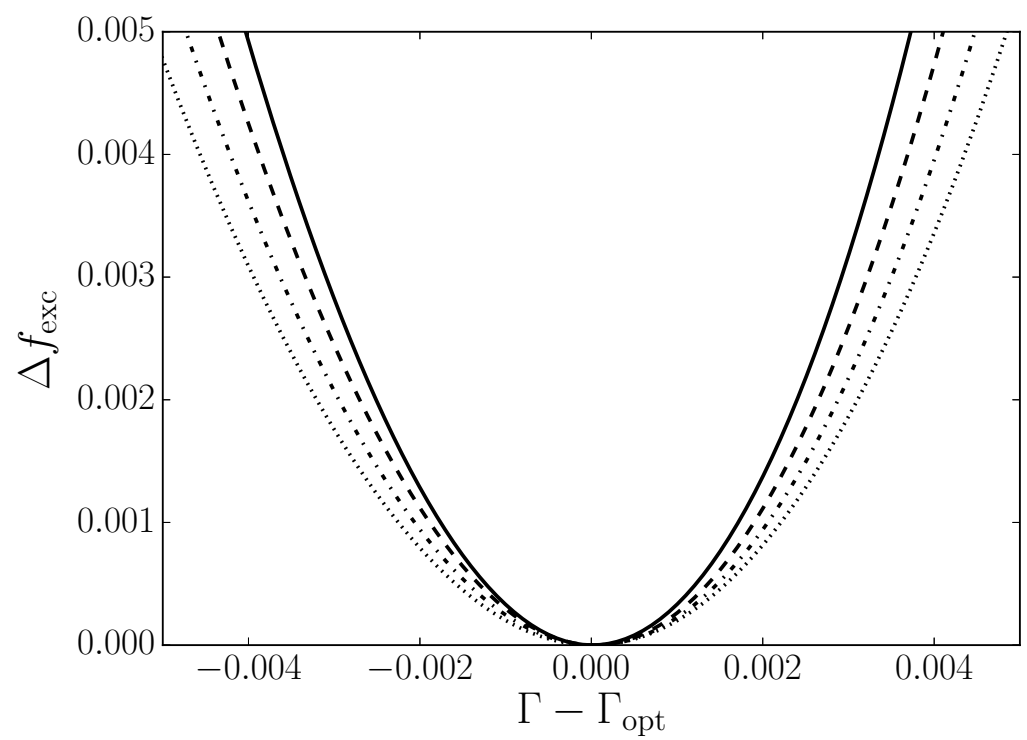

Figure 3: Model free energy density as a function of surface coverage relative to its optimal value $\Gamma_{\mathrm{opt}}$-in-plane interaction effect. Bilayer volume fraction $\phi=0.70$. Second virial coefficient $b_{2}$, from higher to lower bilayer in-plane cohesion: -31 (solid line), -21 (dashed line), -11 (dash-dotted line) and -1 (dotted line). Bending modulus $\kappa=1$., Milner-Roux virial coefficient $\chi=$ 1.55. $\Gamma_{\mathrm{opt}} \approx 0.97$. Free energy densities translated vertically to ensure a value 0 for $\Gamma=\Gamma_{\mathrm{opt}}$

eventually, to a higher surface elasticity.

\subsubsection{Water activity in lamellar stacks of bilayers}

The significance of parameter $\chi$ is most strikingly evidenced when considering the so-called "swelling limit" of the lamellar stack. As long as (direct or effective) inter-bilayer interactions are repulsive enough, adding water to the system will result in swelling the structure: $\ell(\phi)$ increases with decreasing $\phi$. This was indeed the case for the parameters chosen in drawing Fig. 2, again referring to the " $\ell-\Gamma$ " interplay. Conversely, for too weak inter-bilayer repulsions, or in the presence of strong enough attractions, a swelling limit may be reached: Any amount of water added to the lamellar stack above this limit does not incorporate within the structure but phase-separates instead as excess water. A quite interesting property from a theoretical point of view is the presence of a so-called "unbinding" transition [34, 37, 38, occurring at a finite $\chi_{u}$ : For unbound lamellar systems, i.e. $\chi<\chi_{u}, \ell \rightarrow+\infty$ upon swelling, but $\ell$ cannot exceed a limiting value $\ell_{\mathrm{MAX}}$ (and excess water is expelled from the "bound" 
lamellar structure at hydration $\phi_{\min }$ ) if $\chi>\chi_{u}$.

For analysing the occurrence of a "bound" system, osmotic pressure $\Pi_{o}$ is a relevant tool since $\Pi_{o}=0$ when the swelling limit is reached. From the general thermodynamic relation

$$
\Pi_{o}=\phi \frac{\partial f_{\mathrm{exc}}}{\partial \phi}-f_{\mathrm{exc}}
$$

osmotic pressure is straightforwardly expressed from eq. (9) as

$$
\Pi_{o}=\frac{3 \pi^{2}}{64 \kappa} \frac{\phi^{3}}{(\Gamma-\phi)^{3}}-\chi \phi^{2}
$$

Fig. 4 illustrates the control that $\chi$ exerts on the dilution limit. Taking into

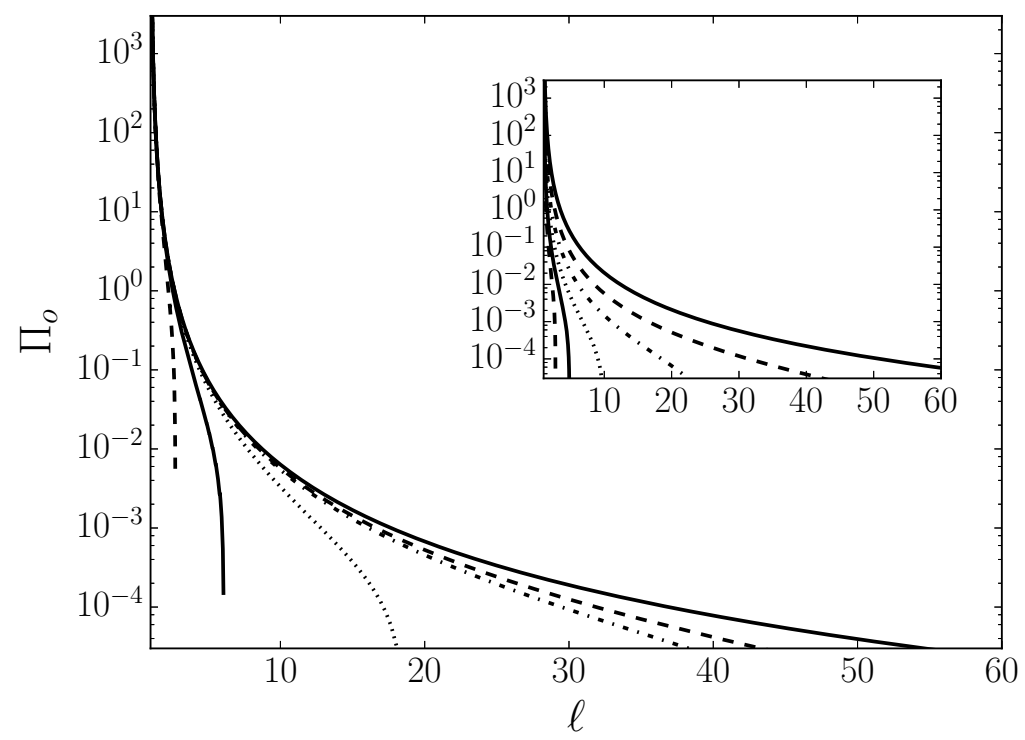

Figure 4: Osmotic pressure equation of state expressed as a function of the stacking period $\ell$. MiLner-Roux virial parameter $\chi$, from weaker to stronger inter-bilayer attractive interactions: 0 (upper solid line), 0.1 (upper dashed line), 0.15 (dash-dotted line), 0.5 (dotted line), 2. (lower solid line) and 8. (lower dashed line). With the values chosen for parameters $\kappa$ and $b_{2}$ (respectively, 0.1 and -11.), $\chi=0.15$ is close to, but greater than $\chi_{u}$ where unbinding occurs, and the swelling limit is $\phi_{\min } \approx 1.4 \%$. Inset: $\chi=0.11, b_{2}=-11$., bilayer bending modulus $\kappa$ : 0.03 (upper solid line), 0.1 (upper dashed line), 0.3 (dash-dotted line, "bound" system with swelling limit $\left.\ell_{\mathrm{MAX}} \approx 26.6\right), 1$. (dotted line), 3. (lower solid line) and 10. (lower dashed line)

account the overall trend favouring swelling that originates in the HELFRICH 
term-the first term in the right-hand side of eq. (11)-, weaker attractive interactions between stacked bilayers, viz. smaller (positive) values for the MiLnERRoux virial parameter $\chi$ lead to systems able to incorporate more water (and even an infinite amount in the case of "unbound" systems).

The unbinding transition may of course also be reached by tuning effective repulsions, that is to say varying $\kappa$, instead of playing with direct interactions through parameter $\chi$, see inset in Fig. 4

An other feature of Fig. 4 is worth mentioning here: In the strongly dehydrated limit where $\phi$ is close to 1 , with therefore $\Gamma_{\mathrm{opt}} \rightarrow 1$ (as suggested in Fig. 2) and $\ell \rightarrow 1$, the dependence of osmotic pressure on $\ell$ is close to being exponential. The model, eq. (9), is thus able to reproduce a property first experimentally established in pioneering reports, Ref. [16, 26, and often discussed in terms of "hydration interactions" between surfactant bilayers (see, for instance, Ref. [27,31,39]). In the present model, however, no such ad hoc interactions have been taken into consideration: Eq. (5), (6), as well as (7) incorporate only fundamental thermodynamic ingredients without postulating any specific profile, e.g. exponential, for inter-bilayer interactions. The physical reality of the so-called "hydration interactions" may therefore be considered as highly dubious [30.

\subsection{Phase diagrams}

As recalled in Section 1 the CBF-to-NBF transition is a kind of spinodal decomposition, the instability being driven within the framework of VRIJ's analysis by diverging "peristaltic" fluctuations. An important question is whether a similar instability actually occurs in the model, eq. (91). The competition in terms of the swelling properties between $\kappa$ and $\chi$ would then not only lead in some cases to the "unbinding" transition discussed in Section 2.1.2 above, that is to say to a lamellar phase - solvent phase separation but also, in other regions of the parameter space, to a lamellar-lamellar phase equilibrium. Such phase separations, more frequent in ternary or higher multi-component systems of stacked bilayers, are indeed sometimes observed experimentally in a few binary lyotropic lamellar phases 40 44.

The so-called "peristaltic" fluctuations in the thin liquid film context, associated to fluctuations in the thickness of the water channel separating the two facing, surfactant-covered air-water interfaces, should not be confused with homonym fluctuations in the context of lyotropic lamellar phases, however. Presumably because the focus is on bilayer objects in the latter case, the "membrane peristaltic" modes, according to the terminology found in Ref. [45, refer to fluctuations in surface coverage $\Gamma$, the fluctuations in water channel thickness giving rise to distinct, "baroclinic" modes. Notwithstanding a perhaps confusing terminology, and similarly to (thin films) "peristaltic" fluctuations, "baroclinic" fluctuations may be divergent in lyotropic lamellar phases. When this is the case, a lamellar-lamellar phase separation occurs as a consequence of the associated spinodal instability.

The criterion for spinodal instability is, at least formally, rather easily established. For a lamellar stack with an overall bilayer content given by $\phi$, 
and therefore a surface coverage $\Gamma_{\text {opt }}$ deduced from eq. (4), local fluctuations $\phi+\delta \phi(\mathbf{x})$ and $\Gamma_{\mathrm{opt}}+\delta \Gamma(\mathbf{x})$ in composition and surface coverage, respectively, are randomly generated by thermal motion within a (macroscopically) infinitesimal volume $\delta V$ located at position $\mathbf{x}$ within the system. Using Boltzmann statistics, such fluctuations occur with a probability $p$ proportional to

$\exp \left\{-\frac{\delta V f_{\mathrm{exc}}\left[\phi+\delta \phi(\mathbf{x}), \Gamma_{\mathrm{opt}}+\delta \Gamma(\mathbf{x})\right]-\mu_{w}\left[N_{w}+\delta N_{w}(\mathbf{x})\right]-\mu[N+\delta N(\mathbf{x})]}{k_{B} T}\right\}$

since the (macroscopic) volume outside $\delta V$ plays the role of a reservoir with chemical potentials $\mu$ and $\mu_{w}$ for surfactant and solvent, respectively. Owing to incompressibility, composition fluctuations inside $\delta V$ correspond to variations in the numbers of solvent $\left(N_{w}\right)$ and surfactant $(N)$ molecules with opposite sign: $v_{w} \delta N_{w}(\mathbf{x})+v \delta N(\mathbf{x})=0, v_{w}$ being the solvent molecular volume, which implies $\delta \phi(\mathbf{x}) \propto \delta N(\mathbf{x})$. For ensuring that the overall composition is indeed given by volume fraction $\phi$, the chemical potentials should satisfy

$$
\frac{\mu}{v}-\frac{\mu_{w}}{v_{w}}=\partial f_{\text {exc }} / \partial \phi
$$

and eq. (4) is of course recovered for ensuring that the surface coverage is $\Gamma_{\mathrm{opt}}$.

For small enough fluctuations, the probability $p$ reduces to

$$
p \propto \exp \left\{-\frac{\frac{\partial^{2} f_{\text {exc }}}{\partial \phi^{2}} \delta \phi^{2}+2 \frac{\partial^{2} f_{\text {exc }}}{\partial \phi \Gamma \Gamma} \delta \phi \delta \Gamma+\frac{\partial^{2} f_{\text {exc }}}{\partial \Gamma^{2}} \delta \Gamma^{2}}{2 k_{B} T} \delta V\right\}
$$

truncating the Taylor expansion to second order. Stability of the homogeneous state requires $p[\delta \phi(\mathbf{x}), \delta \Gamma(\mathbf{x})]$ to be smaller than $p[\delta \phi(\mathbf{x})=0, \delta \Gamma(\mathbf{x})=0]$ for any fluctuations $[\delta \phi(\mathbf{x}), \delta \Gamma(\mathbf{x})]$ or, equivalently

$$
\left\{\begin{array}{l}
0<\frac{\partial^{2} f_{\mathrm{exc}}}{\partial \phi^{2}} \\
0<\frac{\partial^{2} f_{\mathrm{exc}}}{\partial \phi^{2}} \frac{\partial^{2} f_{\mathrm{exc}}}{\partial \Gamma^{2}}-\left(\frac{\partial^{2} f_{\mathrm{exc}}}{\partial \phi \partial \Gamma}\right)^{2}
\end{array}\right.
$$

Conversely, a spinodal decomposition and, ultimately, a lamellar-lamellar phase separation occur if one (or both) inequalities are violated.

A somehow similar discussion has been outlined before, in particular in the more complicated case of three-component (polymer-doped) lamellar phases [46[48. Much more recently, spinodal instabilities in two-component lyotropic lamellar systems have been investigated quite in depth, however [49]. Considering simultaneously the spinodal instability criteria, eq. (15), and the condition for unbound swelling $\Pi_{o}>0$, core features of the phase diagram of binary lamellar stacks described by eq. (9) can be established, as in the examples displayed in Fig. $5 \mathrm{a}$ and $5 \mathrm{~b}$, illustrating $\chi$ and $\kappa$ effects, respectively.

In order to actually describe the lamellar-lamellar phase separation that is bound to occur as the outcome of the spinodal decomposition and, therefore, 


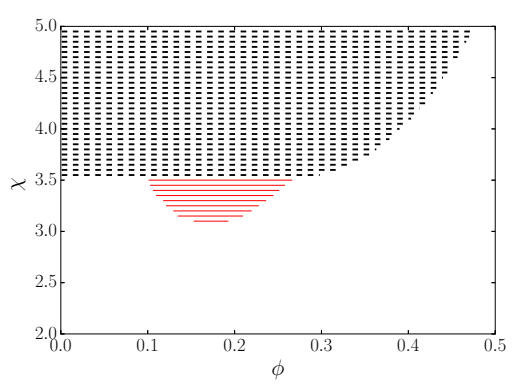

(a) $\phi-\chi$ projection

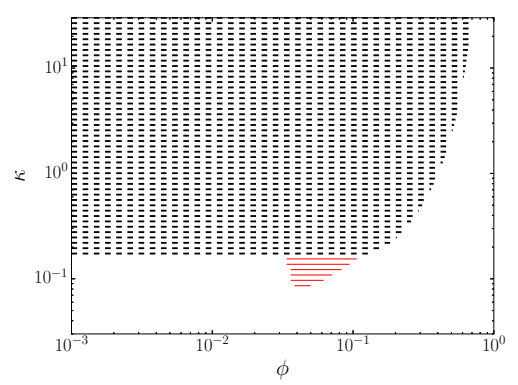

(b) $\phi-\kappa$ projection

Figure 5: Schematic phase diagrams resulting from the model, eq. (9). The black, dashed regions are obtained in analysing the coexistence between pure solvent, $\phi=0$, and a bound lamellar phase with composition $\phi_{\min }$, where $\Pi_{o}\left(\phi_{\min }\right)=0$. The red lines mark spinodal instability regions where $\frac{\partial^{2} f_{\text {exc }}}{\partial \phi^{2}} \frac{\partial^{2} f_{\text {exc }}}{\partial \Gamma^{2}}-\left(\frac{\partial^{2} f_{\text {exc }}}{\partial \phi \partial \Gamma}\right)^{2}<0$. The phase boundaries for lamellar-lamellar phase separations enclose the red-marked regions, that end at critical points. (a) Projection in the $\phi-\chi$ plane, with $\kappa=1$. and $b_{2}=-0.25$. For $\chi$ smaller than $\chi_{u} \approx 3.53$, the lamellar phase is unbound. The swelling limit $\phi_{\min }$ of bound systems increases with MiLner-Roux virial coefficient $\chi$. Critical point coordinates $\chi_{c} \approx 3.067, \phi_{c} \approx 0.162$. (b) Projection in the $\phi-\kappa$ plane, with $\chi=3$. and $b_{2}=-5.0$. For $\kappa$ smaller than $\kappa_{u} \approx 0.2$, the lamellar phase is unbound. The swelling limit $\phi_{\min }$ of bound systems increases with bilayer bending modulus $\kappa$. Critical point coordinates $\kappa_{c} \approx 0.0802, \phi_{c} \approx 0.0415$

to build less schematic phase diagrams than displayed in Fig. 5 , the free energy associated to two coexisting lamellar phases, i.e.

$$
F_{2} \propto \varphi f_{\text {exc }}\left(\phi_{1}, \Gamma_{1}\right)+(1-\varphi) f_{\text {exc }}\left(\frac{\phi-\varphi \phi_{1}}{1-\varphi}, \Gamma_{2}\right)
$$

where $\left\{\phi_{1}, \Gamma_{1}\right\}$ and $\left\{\phi_{2} \equiv\left(\phi-\varphi \phi_{1}\right) /(1-\varphi), \Gamma_{2}\right\}$ are the respective compositions variables for phases 1 and $2, \phi$ is the overall surfactant volume fraction, and $\varphi$ the fraction of the total volume occupied by phase 1 , has to be minimised with respect to all the fluctuating variables, namely $\Gamma_{1}, \Gamma_{2}, \phi_{1}$ and $\varphi$. The procedure formally leads to two equations similar to eq. (4), as well as two equations stipulating osmotic equilibrium between the two phases, namely equal surfactant chemical potentials and equal osmotic pressures (or solvent chemical potentials). With the lever rule $\varphi \phi_{1}+(1-\varphi) \phi_{2}=\phi$ implicit, the two latter equations are commonly cast in the following form

$$
\begin{aligned}
\left.\frac{\partial f_{\text {exc }}}{\partial \phi}\right|_{\left\{\phi_{1}, \Gamma_{1}\right\}} & =\left.\frac{\partial f_{\text {exc }}}{\partial \phi}\right|_{\left\{\phi_{2}, \Gamma_{2}\right\}} \\
f_{\text {exc }}\left(\phi_{1}, \Gamma_{1}\right) & =f_{\text {exc }}\left(\phi_{2}, \Gamma_{2}\right)+\left.\left(\phi_{1}-\phi_{2}\right) \frac{\partial f_{\text {exc }}}{\partial \phi}\right|_{\left\{\phi_{2}, \Gamma_{2}\right\}}
\end{aligned}
$$


that would be geometrically interpreted as the "common tangent construct" in the classical problem of a liquid-liquid phase separation in binary solutions.

In practice, considerable efforts have to be spent for explicitly minimising eq. (16) and eventually get phase boundaries $\phi_{1}$ and $\phi_{2}$, as well as surface coverages $\Gamma_{\mathrm{opt}}\left(\phi_{1}\right)$ and $\Gamma_{\mathrm{opt}}\left(\phi_{2}\right)$. The problem is indeed more difficult than separately solving for the volume fraction $\phi$ the equations $\Pi_{o}=0, \partial^{2} f_{\text {exc }} / \partial \phi^{2}=$ 0 and $\partial^{2} f_{\text {exc }} / \partial \phi^{2} \times \partial^{2} f_{\text {exc }} / \partial \Gamma^{2}-\left(\partial^{2} f_{\text {exc }} / \partial \phi \partial \Gamma\right)^{2}=0$. Fig. 6 gives an illustration of the simultaneous solution for two volume fractions of eq. (17) in terms of osmotic pressure and surfactant chemical potential.
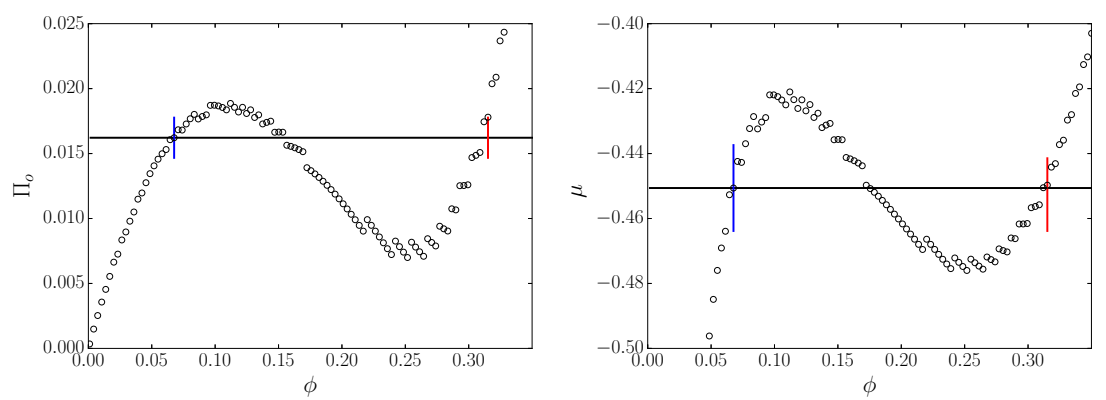

Figure 6: Osmotic pressure $\Pi_{o}$ and surfactant chemical potential $\mu$ vs. composition $\phi$ according to numerical estimates from the model, eq. (9). Parameters: $\kappa=1 ., b_{2}=-0.25, \chi=3.4$. A phase separation occurs between a dilute lamellar phase with composition $\phi_{\text {dil }} \approx 6.8 \%$ (vertical blue line. Stacking period $\ell_{\text {dil }} \approx 3.4$ ) and a concentrated one with $\phi_{\text {conc }} \approx 31.2 \%$ (vertical red line. Stacking period $\ell_{\text {conc }} \approx 2.1$ )

Though a complete study is left to undaunted readers, a phase diagram less schematic than in Fig. 5 a is nevertheless displayed for illustration purposes in Fig. 7 It is interesting to observe that, for this specific case, the unbinding transition no longer occurs: For large enough interactions between surfactant bilayers-as described by parameter $\chi^{-}$, hydrating the lamellar stack leads to a lamellar-lamellar phase separation, the dilute lamellar phase being unbound. Below a critical value $\chi_{c}$ (close to 3.067 in the present case), the lamellar phase can be continuously swollen by water until infinite dilution. A decade-old controversy about swelling of lecithin-based lamellar phases [16,32, qualitatively at least, might be settled by the above-described feature.

\section{Newton black films}

\subsection{Thermodynamic considerations}

Considering the structural analogy between soap films and lyotropic lamellar phases, the ideas leading to the model free energy for stacked bilayers, eq. (9), 


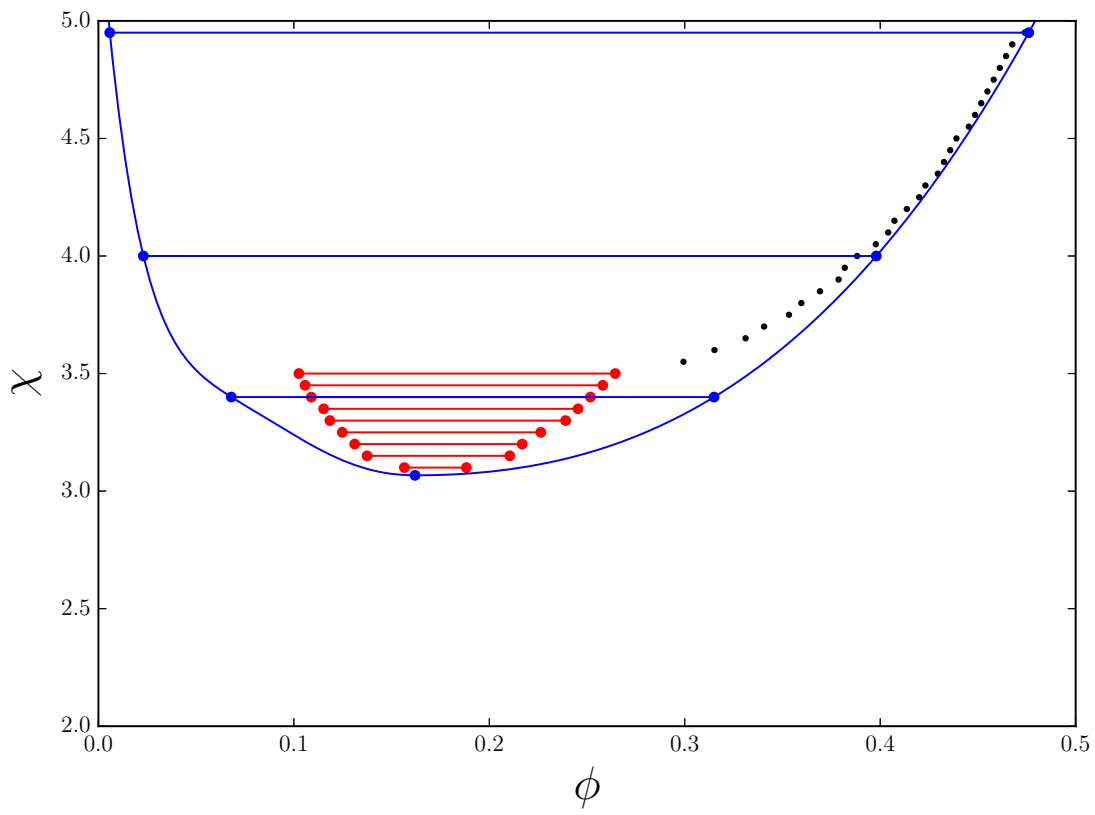

Figure 7: Projection in the $\phi-\chi$ plane, with $\kappa=1$. and $b_{2}=-0.25$ of the phase diagram resulting from the model, eq. (9). The black circles mark loci where the osmotic pressure $\Pi_{o}(\phi)$ first vanishes when increasing hydration. The red circles mark loci where $\Delta \equiv \frac{\partial^{2} f_{\mathrm{exc}}}{\partial \phi^{2}} \frac{\partial^{2} f_{\mathrm{exc}}}{\partial \Gamma^{2}}-\left(\frac{\partial^{2} f_{\mathrm{exc}}}{\partial \phi \partial \Gamma}\right)^{2}$ vanishes, and $\Delta$ is negative within the region delimited by the red lines. The phase boundaries for lamellar-lamellar phase separations are marked by blue circles, solving eq. (17), with interpolated blue lines. Black circles being within the blue-enclosed region, unbinding is here circumvented by a lamellar-lamellar phase separation

may be used almost directly for thermodynamic models of soap films and, more specifically, of Newton black films. The composition variable $\phi$ now refers to the amount of "solute" in the aqueous channel, $\ell$ being related to its thickness, while $\Gamma$ remains the number of surfactant molecules per unit monolayer area. At contrast with the case of stacked bilayers, i) the exchange of surfactant molecules between the two monolayers and the aqueous channel is explicitly taken into account now, and ii) the meaning of the "swelling law" has to be reinterpreted. Assuming homogeneous and ideally flat, parallel monolayers, that is to say choosing a "film model" according to the terminology of Ref. [50], a simple geometric description of a volume $V \equiv \ell \mathcal{A}=N_{w} v_{w}+N_{\text {tot }} v$ with $N$, respectively $N_{\text {tot }}-N$, surfactant molecules covering two monolayers with area $\mathcal{A}$, resp. dispersed in the aqueous channel, gives

$$
\phi=\frac{\phi_{0}-2 \frac{v}{\ell} \Gamma}{1-2 \frac{v}{\ell} \Gamma}
$$


with $\phi_{0}=N_{\text {tot }} v /\left(N_{w} v_{w}+N_{\text {tot }} v\right)$ the overall system composition. For an easier comparison with eq. (2), an equivalent form of such a geometric "dilution law" is

$$
\ell \frac{\phi_{0}-\phi}{1-\phi}=2 v \Gamma
$$

As it appears below, the " $\ell-\Gamma$ " interplay in soap films is much less intuitive than in lamellar phases, the dilution law being more intricate.

An important difference between (macroscopic) soap films and Newton black films should be mentioned here: For macroscopic soap films, at the water-air interface of a Langmuir trough for instance, there is no thinning and $\ell$ is fixed, usually to a value much larger than any molecular scales. At contrast, both $\ell$ and $\phi($ or $\Gamma)$ are fluctuating quantities in Newton black films, $\ell$ moreover being in the order of a molecular scale. The thickness $h$ of the aqueous channel may be defined such that $h \mathcal{A}=N_{w} v_{w}+\left(N_{\text {tot }}-N\right) v$, and it is also a fluctuating quantity, directly linked to monolayer coverage $\Gamma$ :

$$
h=\ell-2 v \Gamma
$$

The so-called "thick limit"-without thinning-thus describes, e.g. Langmuir troughs, and thinning is a characteristic feature of (common or Newton) black films.

A legitimate purpose of any thermodynamic model of soap or Newton black films is, given the amount of surface-active material $\phi_{0}$ and, if appropriate, the film aspect ratio $\ell$, to build equations of state for surfactant remaining dispersed within the aqueous channel or sub-phase, $\phi$, and film coverage, $\Gamma$, as well as to describe surface tension $\gamma$, surface dilational modulus $E$, etc. If thinning of the (black) film by border capillary suction is taken into account, predicting the optimal aspect ratio $\ell_{\text {opt }}$ is also, of course, one of the quite important targets of the model. Such approaches were attempted, and fulfilled, many times along the years, with various viewpoints, see for instance [29, 35, 36, 51, 52,

A convenient starting point is the Gibbs free energy with the following form

$$
G=G_{\text {channel }}\left(N_{w}, N_{\text {tot }}-N\right)+\mathcal{A} g_{\text {film }}(\Gamma, h)
$$

with $G_{\text {channel }}$ the Gibbs free energy for the species dispersed within the aqueous channel, $g_{\text {film }}$ the Gibbs free energy (surface) density for the two surfactantcovered monolayers facing each other across the aqueous channel and, of course, $N=2 \Gamma \mathcal{A}[53]$. The $h$ dependence in the surface contribution is meant to account for film-film interactions ("disjoining pressure") across the aqueous channel that cannot be neglected for Newton black films.

Osmotic equilibrium for the surfactant that exchanges between monolayers and the aqueous channel requires

$$
-\mu+\frac{1}{2}\left[\frac{\partial g_{\mathrm{film}}}{\partial \Gamma}-2 v \frac{\partial g_{\mathrm{film}}}{\partial h}\right]=0
$$

a minimisation equation somehow similar to eq. (4), Section 2.1, where $\mu$ is the surfactant chemical potential in the aqueous channel for composition $\phi$. 
Applying the Gibbs-Duhem relation to the first term in the right-hand side of eq. (21) yields

$$
G=N_{w} \mu_{w}(\phi)+N_{\text {tot }} \mu(\phi)+\mathcal{A}\left[g_{\text {film }}(\Gamma, h)-2 \mu(\phi) \Gamma\right]
$$

with therefore the surface tension of the film, defined by the area-dependent term in the Gibbs free energy, given by:

$$
\gamma=g_{\text {film }}(\Gamma, h)-2 \mu(\phi) \Gamma
$$

If thinning is taken into account, $\ell$ is also a fluctuating quantity as mentioned above and a mechanical equilibrium condition has to be considered in addition to the osmotic equilibrium condition, eq. (22). In the so-called "thin film pressure balance" set-up (see Ref. 54] and, more recently, e.g., Ref. [55]), the hydrostatic pressure within the film, $p_{i}$, differs from the pressure in the outside medium, $p_{e}$. This amounts to adding a pressure term $\left[\left(p_{e}-p_{i}\right) \mathcal{A}\right] \times \ell$ to the right-hand sides of eq. (21) or (23), see Ref. [50]. Mechanical equilibrium of the film then requires

$$
\ell\left(\frac{\partial g_{\mathrm{film}}}{\partial h}+p_{e}-p_{i}\right)=g_{\mathrm{film}}-2 \mu \Gamma
$$

In the "thick film" limit, the difference between $\phi$ and $\phi_{0}$ disappears and the "disjoining pressure" contribution to the film surface tension, though essential for ensuring an actual thinning of freely-suspended black films, becomes meaningless. The classical relation

$$
\frac{\partial \gamma}{\partial \ln \phi}=k_{B} T \times 2 \Gamma
$$

is then recovered (with a factor 2 because the film comprises two facing monolayers) by differentiating eq. (24), if it can be assumed that the surfactant solution behaves ideally in the aqueous sub-phase.

Global stability of the (macroscopic) soap film-i.e. not yet considering local "peristaltic" fluctuations-can be studied by expanding the Gibbs free energy, eq. (21), to second order with respect to fluctuations $\delta \phi$ in aqueous channel composition or, equivalently, fluctuations $\delta \Gamma$ in surface coverage, both originating in $\delta N$ fluctuations. In terms of surface coverage fluctuations, the result is

$$
G \approx G_{\mathrm{opt}}+\frac{\mathcal{A}}{2}\left[-\frac{\partial^{2} f_{\mathrm{exc}}}{\partial \phi^{2}} \frac{4 v^{2}(1-\phi)^{2}}{\ell\left(1-\frac{2 v}{\ell} \Gamma\right)}+\frac{\partial^{2} g_{\mathrm{film}}}{\partial \Gamma^{2}}-4 v \frac{\partial^{2} g_{\mathrm{film}}}{\partial \Gamma \partial h}+4 v^{2} \frac{\partial^{2} g_{\mathrm{film}}}{\partial h^{2}}\right] \delta \Gamma^{2}
$$

Global stability is thus ensured when the expression within square brackets is positive. As a matter of fact, it is closely related to the surface dilational modulus $E$, usually defined by

$$
E \equiv-\Gamma \frac{\delta \gamma}{\delta \Gamma}
$$

with however an ambiguity (also encountered when defining heat capacities, for instance) regarding the mechanism for the infinitesimal variation $\delta \Gamma$ in surface 
coverage. Here, the dilational modulus is defined for fixed geometric film parameters $V \equiv N_{w} v_{w}+N_{\text {tot }} v$ (incompressibility) and $\mathcal{A}$ (no thinning), and is therefore given by

$$
E=\left[\frac{\partial^{2} g_{\text {film }}}{\partial \Gamma^{2}}-4 v \frac{\partial^{2} g_{\text {film }}}{\partial \Gamma \partial h}+4 v^{2} \frac{\partial^{2} g_{\text {film }}}{\partial h^{2}}\right] \Gamma^{2}
$$

that is to say without the osmotic compressibility component $\partial^{2} f_{\text {exc }} / \partial \phi^{2}$ that appears in eq. (27).

As an illustration, optimal surface coverage, $\Gamma_{\mathrm{opt}}$, surface tension $\gamma$ and surface dilational modulus $E$ are plotted in Fig. 8 as functions of composition $\phi_{0}$ in the aqueous channel, with a standard model for the chemical potential $\mu$-ideal solution: $f_{\text {exc }}=k_{B} T[(1-\phi) \ln (1-\phi)+\phi \ln \phi] / v$-and a Gibbs free energy surface density $g_{\text {film }}$ similar in spirit to eq. (7), but without any "disjoining pressure" contribution: $g_{\text {film }}=\gamma_{0}+k_{B} T\left\{\Gamma \ln \left[\Gamma /\left(\Gamma_{\infty}-\Gamma\right)\right]+b_{2} \Gamma^{2} / 2-\chi \Gamma\right\}$, where $\gamma_{0}$ is the water-air surface tension, $\Gamma_{\infty}$ the upper limit for surface coverage, $b_{2}$ a virial coefficient for describing in-plane interactions between adsorbed surfactant molecules and $\chi$ a measure of the adsorption energy per molecule. Fig. 8 gives a (model-dependent) quantitative and explicit basis to the state-
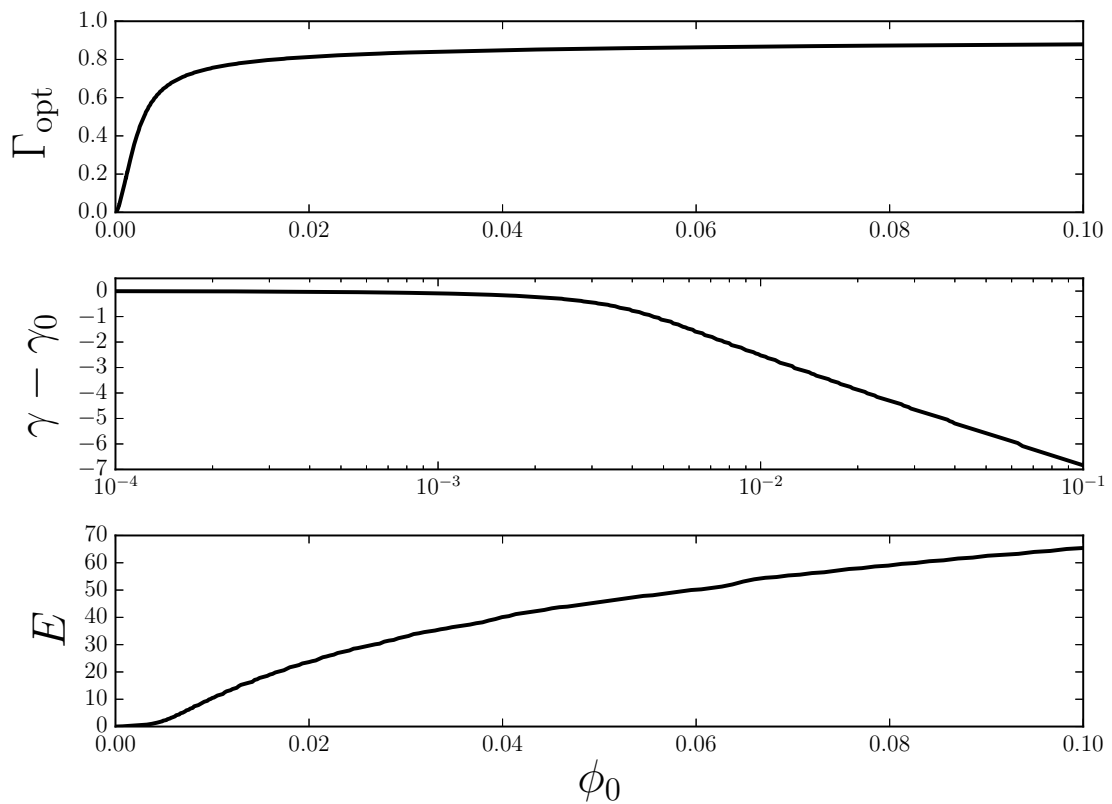

Figure 8: Equations of state in the "thick film" limit for optimal surface coverage $\Gamma_{\mathrm{opt}}$, surface tension $\gamma$ and surface dilational modulus $E$, resulting from eq. (22), eq. (24) and eq. (27), respectively. Reduced units using $\Gamma_{\infty}$ and $k_{B} T \Gamma_{\infty}$ for surface coverage and surface energy, respectively

ment, adapted from Ref. [6]: 
It is also important to note, that since all of the film-stabilisation mechanisms rely on the same fundamental property, surfactant adsorption, they are inherently interdependent.

and, qualitatively at least, describes rather satisfactorily the "thick film" data [6]. Note that taking explicitly into account the formation of surfactant micelles is not necessary to explain a high value for the surface dilational modulus $E$.

\subsection{Peristaltic fluctuations}

The thinning phenomenon of a liquid film obviously requires $\ell$ to vary. Moreover, as recalled in Section 1, the film stability problem once an equilibrium value $\ell_{\text {eq }}$ has been reached should be investigated in the presence of (local) peristaltic fluctuations. Owing to incompressibility, the overall volume $V \equiv \ell \mathcal{A}$ cannot vary: Peristaltic fluctuations $(\delta \ell$ or $\delta h)$ therefore imply area fluctuations $(\delta \mathcal{A})$, with surface tension acting as a "restoring force". In addition, even though the total numbers of solvent $N_{w}$ and surfactant $N_{\text {tot }}$ molecules remain constant, local concentrations and surface coverage fluctuations are allowed and they are, generally speaking, spatially dependent. Osmotic compressibility in the aqueous channel and dilational modulus in the two monolayers act as additional "restoring forces". The various fluctuating fields are coupled, making a detailed analysis somehow involved.

Following VRIJ, the stability analysis is here restricted to the study of symmetric modes with long wavelength and small amplitude [4]. It is also considered that the thinning process has allowed to reach the value $\ell_{\mathrm{eq}}$ for the geometric parameter $\ell$. The film area $V / \ell_{\text {eq }}$ has therefore become a fixed quantity, extremely large, in molecular area units, for real black films. With $t \equiv h / 2$ half the local thickness of the film, a Gibbs free energy generalising eq. (21) and taking into account conservation of matter may be expressed as

$$
\begin{gathered}
G=\int d^{2} x \quad\left\{2 t\left[p+(1-\phi) \frac{\mu_{w}^{+}}{v_{w}}+\phi \frac{\mu^{+}}{v}+f_{\mathrm{exc}}(\phi)\right]\right. \\
\left.+\left[1+\frac{(\nabla t)^{2}}{2}\right] g_{\mathrm{film}}(\Gamma, t)\right\} \\
-\frac{\mu}{v}\left(2 t \phi-\ell \phi_{0}+2 v \Gamma\right) \\
-\frac{\mu_{w}}{v_{w}}\left[2 t(1-\phi)-\ell\left(1-\phi_{0}\right)\right]
\end{gathered}
$$

where $t(\mathbf{x}), \phi(\mathbf{x})$ and $\Gamma(\mathbf{x})$ are now considered as fluctuating fields, $p, \mu_{w}^{+}$and $\mu^{+}$are (constant) pressure and chemical potential reference parameters, and $\mu$ (respectively, $\mu_{w}$ ) is a Lagrange multiplier ensuring the conservation of $N_{\text {tot }}$, resp. $N_{w}$, viz. conservation of the total number of surfactant, resp. solvent, molecules. It is, of course, assumed here that the standard thermodynamic description of the system can be safely extended down to molecular scales.

The 3 Euler-Lagrange equations associated to the fluctuating fields in eq. (30) 
are easily derived:

$$
\begin{aligned}
0= & {\left[1+\frac{(\nabla t)^{2}}{2}\right] \frac{\partial g_{\mathrm{film}}}{\partial \Gamma}-2 \mu } \\
0= & -\frac{\nabla^{2} t}{2} g_{\mathrm{film}}+\left[1+\frac{(\nabla t)^{2}}{2}\right] \frac{\partial g_{\mathrm{film}}}{\partial t} \\
& +2\left[p+(1-\phi) \frac{\mu_{w}^{+}-\mu_{w}}{v_{w}}+\phi \frac{\mu^{+}-\mu}{v}+f_{\mathrm{exc}}(\phi)\right] \\
0= & -\frac{\mu_{w}^{+}-\mu_{w}}{v_{w}}+\frac{\mu^{+}-\mu}{v}+\frac{\partial f_{\mathrm{exc}}}{\partial \phi}
\end{aligned}
$$

with two conservation laws associated to the two Lagrange multipliers

$$
\begin{aligned}
& 0=\int d^{2} x\left[2 t \phi-\ell \phi_{0}+2 v \Gamma\right] \\
& 0=\int d^{2} x\left[2 t(1-\phi)-\ell\left(1-\phi_{0}\right)\right]
\end{aligned}
$$

As expected, constant channel composition $\phi$ and thickness $h$ verifying eq. (18) and eq. (20) are possible solutions of eq. (31) with a constant surface coverage $\Gamma$, and eq. (22) is recovered for such constant "fields".

The second order expansion of the Gibbs free energy, eq. (30), with peristaltic fluctuations about the constant solution of the Euler-Lagrange equations explicitly taken into account, readily leads to

$$
\begin{aligned}
\delta G \approx \frac{1}{2} \int d^{2} x \quad & {\left[2 t \frac{\partial^{2} f_{\text {exc }}}{\partial \phi^{2}} \delta \phi^{2}+(\nabla \delta t)^{2} g_{\mathrm{film}}\right.} \\
& \left.+\frac{\partial^{2} g_{\mathrm{film}}}{\partial \Gamma^{2}} \delta \Gamma^{2}+2 \frac{\partial^{2} g_{\mathrm{film}}}{\partial \Gamma \partial t} \delta \Gamma \delta t+\frac{\partial^{2} g_{\mathrm{film}}}{\partial t^{2}} \delta t^{2}\right]
\end{aligned}
$$

a relation more conveniently expressed in Fourier space owing to Parseval's identity, introducing mode expansions of the fluctuating fields

$$
\psi(\mathbf{x})=\sum_{\mathbf{k}} \psi_{\mathbf{k}} e^{i \mathbf{k} \cdot \mathbf{x}}
$$

as

$$
\begin{aligned}
\delta G=\frac{\mathcal{A}}{2} \sum_{\mathbf{k}} \quad & {\left[2 t \frac{\partial^{2} f_{\mathrm{exc}}}{\partial \phi^{2}} \phi_{\mathbf{k}} \phi_{-\mathbf{k}}\right.} \\
& +\left(\frac{\partial^{2} g_{\mathrm{iflm}}}{\partial t^{2}}+k^{2} g_{\mathrm{film}}\right) t_{\mathbf{k}} t_{-\mathbf{k}} \\
& \left.+2 \frac{\partial^{2} g_{\mathrm{film}}}{\partial \Gamma \partial t} \Gamma_{\mathbf{k}} t_{-\mathbf{k}}+\frac{\partial^{2} f_{\mathrm{film}}}{\partial \Gamma^{2}} \Gamma_{\mathbf{k}} \Gamma_{-\mathbf{k}}\right]
\end{aligned}
$$

Quite similarly to Section 2.2, the stability of the film with respect to diverging fluctuations requires characteristic inequalities to be fulfilled, namely

$$
\left\{\begin{aligned}
0 & <\frac{\partial^{2} g_{\mathrm{film}}}{\partial \Gamma^{2}} \\
0 & <\frac{\partial^{2} f_{\text {exc }}}{\partial \phi^{2}} \\
0 & <\frac{\partial^{2} g_{\mathrm{film}}}{\partial \Gamma^{2}}\left(\frac{\partial^{2} g_{\mathrm{film}}}{\partial t^{2}}+k^{2} g_{\mathrm{film}}\right)-\left(\frac{\partial^{2} g_{\mathrm{film}}}{\partial \Gamma \partial t}\right)^{2}
\end{aligned}\right.
$$

Conversely, if

$$
\Delta \equiv \frac{\partial^{2} g_{\mathrm{film}}}{\partial \Gamma^{2}} \frac{\partial^{2} g_{\mathrm{film}}}{\partial t^{2}}-\left(\frac{\partial^{2} g_{\mathrm{film}}}{\partial \Gamma \partial t}\right)^{2}
$$


is negative, any mode with a wavelength larger than the critical wavelength

$$
\lambda_{c}=2 \pi \sqrt{\frac{g_{\mathrm{film}} \frac{\partial^{2} g_{\mathrm{film}}}{\partial \Gamma^{2}}}{-\Delta}}
$$

is bound to grow without limit. As was the case for the lamellar phase stability problem, eq. (15) in Section 2.2, film stability thus appears to be driven by an interplay between lateral compressibility and "dilution" properties, the latter again depending on interactions between surfactant-covered interfaces across aqueous channels, eq. (25).

It is interesting to observe that the stability criterion obtained here is not exactly equivalent to VRIJ's criterion, even in the special limit where the abovementioned interplay between lateral compressibility and "dilution" properties does not exist because the cross-term $\partial^{2} g_{\text {film }} / \partial \Gamma \partial t$ is equal to 0 . Indeed, the factor in front of $k^{2}$ in the last condition, eq. (36), is not the surface tension $\gamma$, eq. (24), but the Gibbs free energy (surface) density $g_{\text {film }}$, which results in a slightly different equation for the critical wavelength, eq. (38), as compared to Ref. [4].

To proceed further, explicit models for $g_{\text {film }}(\Gamma, h)$ should be considered. As has been done for lamellar stacks of bilayers in Section 2.1, quite a common approach amounts to considering separately the "lateral" equation of statesurfactant adsorption isotherms-and the "vertical" equation of state ("disjoining pressure") contributions, with in the latter case terms from van der Waals and electrostatic forces usually discussed in details, as for instance by SHELudKo in a classical text, Ref. [56. However, because of eq. (36), "lateral" and "vertical" contributions should be considered simultaneously, at least in principle, as hinted already long ago [5]: On the basis of a numerical study of van der Waals attractions indicating that the Hamaker constant actually depends on surface coverage [57, it was then suggested that the optimal coverage $\Gamma_{\text {opt }}$ not only depends on $\phi_{0}$, but could also depend on film thickness even though any "significant dependence" was ruled out [5].

Unfortunately, physically sound models do not seem to be available yet for Newton black films. For nevertheless illustrating the power of the abovedescribed approach and, hopefully, motivating further research, the study of a "toy" model with a reasonable "lateral" contribution and a (partially) ad hoc "vertical" contribution is proposed below. Specifically

$$
\begin{aligned}
g_{\text {film }}(\Gamma, h)= & \gamma_{0} \\
& +k_{B} T\left\{\Gamma \ln \left[\Gamma /\left(\Gamma_{\infty}-\Gamma\right)\right]+b_{2} \Gamma^{2} / 2-\chi \Gamma\right\} \\
& +k_{B} T \Gamma_{\infty} h_{\min } /\left(h-h_{\min }\right)-V / h^{2}
\end{aligned}
$$

with parameters $h_{\text {min }}$ being the limiting thickness of a wholly dehydrated Newton black film, and $V>0$ a Hamaker-like constant associated to film-film van der Waals interactions across the water channel. While the van der Waals term 
is, of course, extremely well documented and not subject to controversy, it is important to stress here that the repulsive term that diverges at a finite separation $h_{\min }$ in eq. (39) is here introduced $a d$ hoc, not being supported by any physical argument similar to HELFRICH's expression for the undulation interaction in lamellar phases, eq. (5) in Section 2.1. Other choices would be equally acceptable if they implement a very strong repulsion at small separations as, for instance, an adaptation for the one-dimensional geometry relevant here of the repulsive part of a $(6,12)$ LENNARD-JONES potential, yielding a $1 / h^{9}$ term [58]. An electrostatic repulsive contribution should actually be added to the "vertical" component in eq. (39), as it is practically always observed, even with non-ionic surfactants, in disjoining pressure measurements [6, [58, 59].

Fig. 9 gives the results for black film coverage $\Gamma$, surface tension $\gamma-\gamma_{0}$ and thickness $\ell$, as a function of differential pressure $p_{e}-p_{i}$ in the film obtained by minimising the black film Gibbs free energy, eq. (23) with the pressure term included. The grey regions mark the pressure range where peristaltic fluctuations are diverging, i.e. $\Delta<0$ in eq. (37).

It is interesting to note that the optimal surface coverage predicted by the model varies quite significantly, and starts decreasing when enough differential pressure is applied to the film or, in other words, when the film becomes black [60. Such a feature, qualitatively speaking, is not unexpected. Indeed, a $1 \mathrm{~cm}^{3}$ volume of, say, sodium dodecyl sulphate at the critical micellar concentration contains $\mathrm{ca}$. $N_{\text {tot }}=5 \times 10^{18}$ dodecyl sulphate, surface-active ions that have to cover two air-water interfaces of area $\mathcal{A}=2 \times 10^{2} \mathrm{~m}^{2}$ if the film aspect ratio is $\ell=5 \mathrm{~nm}$. The upper limit for surface coverage is therefore $\Gamma_{\max }=1.25 \times 10^{-2} \mathrm{~nm}^{-2}$, nearly 3 orders of magnitude less than the maximum surface coverage in the "thick film" limit, typically a few molecules per squared nanometres [6]. Owing to the osmotic equilibrium between the two surfactantcovered interfaces and aqueous channel, some surface-active molecules have to remain dispersed in the core part of the film, which leads to an optimal coverage even less than $10^{-3} \times \Gamma_{\infty}$. Fig. 10 illustrates this intricacy of the " $\ell-\Gamma$ " interplay, with the channel composition $\phi$ as a third player specific to thinning films, encapsulated by eq. (19). The saturation of $\phi_{\mathrm{opt}}$ to $\phi_{0}$ in the limit of a very high differential pressures, that is to say for a highly dehydrated Newton black film is, presumably, an artefact of the ad hoc component of the "toy" model considered here, as it would amount to unrealistically small optimal surface coverages $\Gamma_{\text {opt }}$ owing to the " $\ell-\Gamma$ " interplay.

The equation of state for the black film "disjoining pressure" (identified to the differential pressure $p_{e}-p_{i}$ applied to it) as a function of the black film aqueous core thickness $h$ is displayed in Fig. 11]. Though using the common terminology is not legitimate here, because there is only one repulsive barrier included in the "toy" model, eq. (39), it is tempting to describe as Newton and, respectively common black films the domains located to the left, resp. to the right, of the (grey) peristaltic instability region. In the (kind of) common black film domain, the "disjoining pressure" decays approximately as $h^{-2}$, viz. varies as the van der Waals contribution to the Gibbs free energy (surface) density in eq. (39). The precise law for its divergence when $h_{\mathrm{opt}} / h_{\min } \rightarrow 1$ obviously 

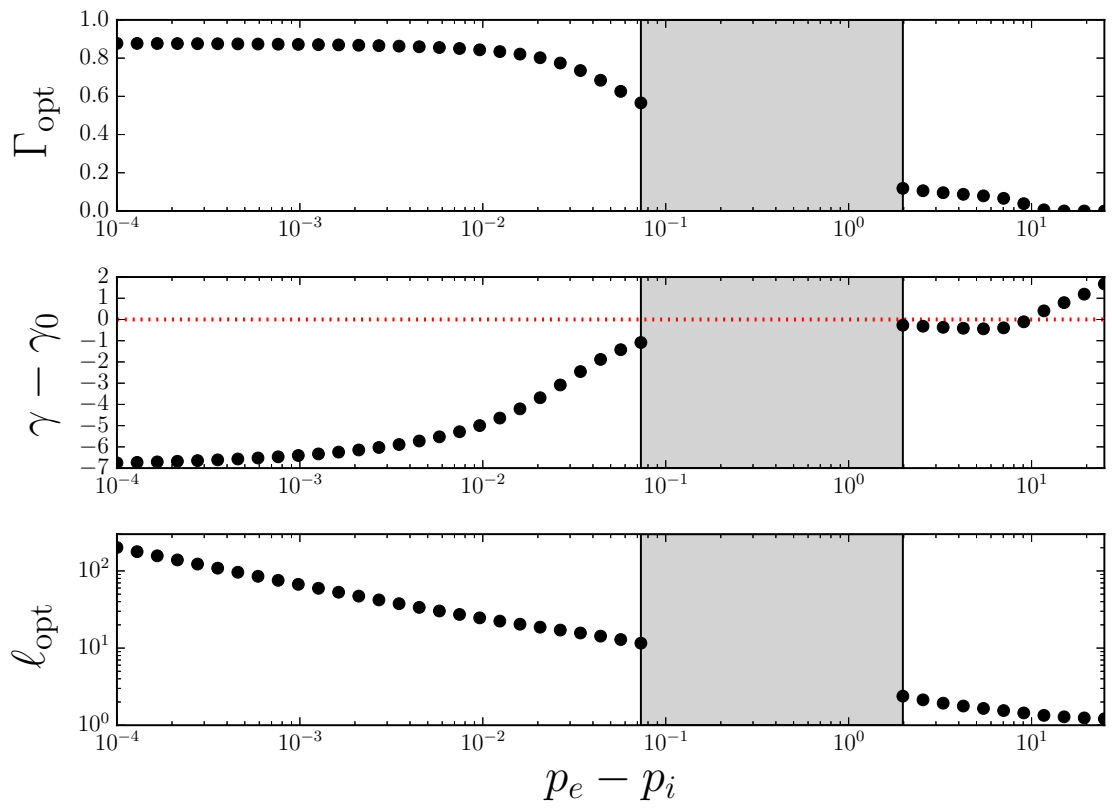

Figure 9: Black film equations of state in reduced units as functions of differential pressure $p_{e}-p_{i}$ for optimal surface coverage $\Gamma_{\mathrm{opt}}$, surface tension $\gamma$ and optimal thickness $\ell_{\mathrm{opt}}$, resulting from eq. (22), (24) and (19), respectively. The high (low) differential pressure range corresponds to a Newton (common) black film. Peristaltic fluctuations are divergent in the cray region. The horizontal dotted red line in the second panel indicates a surface tension equal to the bare water-air surface tension $\gamma_{0}$. Note that optimal surface coverage starts to decrease when approaching the unstable pressure range, and is much lower for Newton black films than for common black films

depends on the ad hoc component of the "toy" model and should therefore not be taken too seriously, even though it resembles, qualitatively at least, to some data found experimentally [21, 59 .

\section{Conclusion}

The structural analogy between soap films, in particular common or Newton black films, and lamellar stacks of surfactant bilayers allows considering these systems with a unified point of view regarding fluctuations and interactions, on one hand, and thermodynamic modelisation on the other hand. Phase diagrams, "vertical" or "lateral" equations of state have been routinely investigated for a long time in the two kinds of systems, and a lingua franca has emerged from the shared problems and objectives in the film and lamellar stack communities. The 


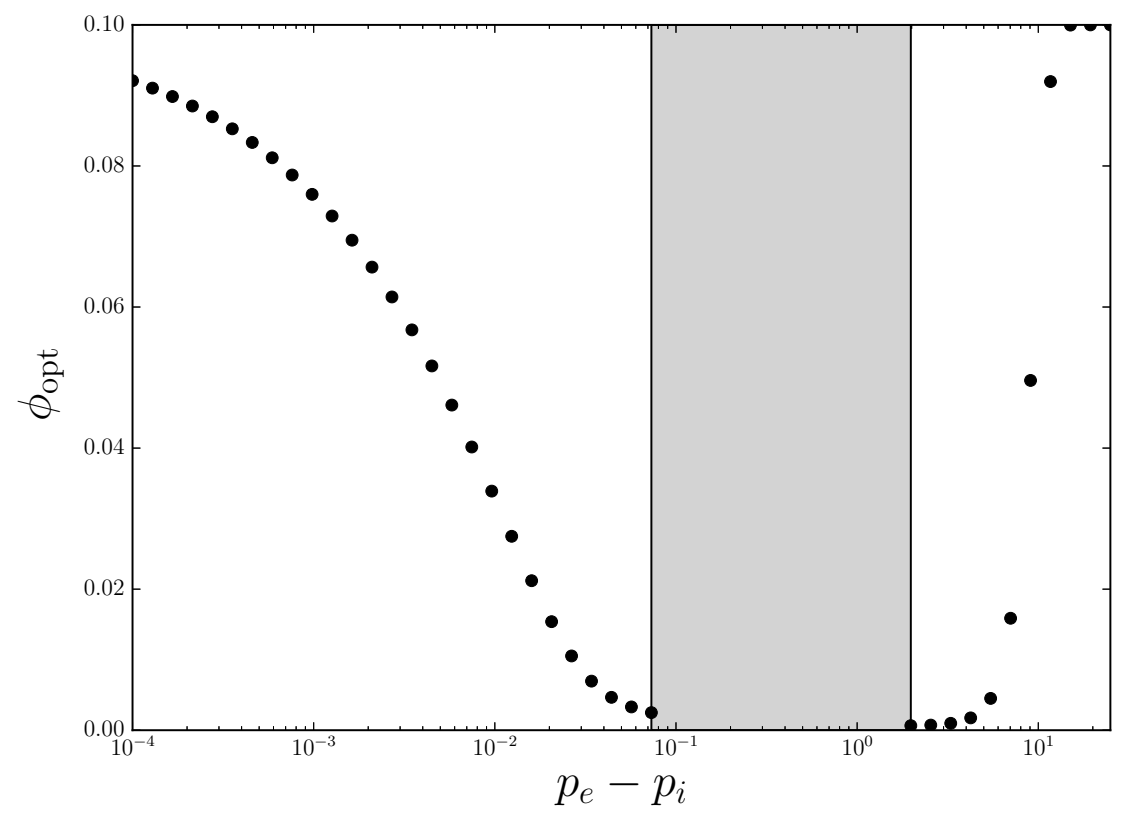

Figure 10: Optimal channel composition $\phi_{\mathrm{opt}}$ as a function of the differential pressure $p_{e}-p_{i}$

elusive nature of the short-range and very strong repulsive interactions between surfactant-covered interfaces, acting across water channels, remains controversial, but both communities, warned long ago by visionary precursors [4, 32], are now firmly convinced that fluctuations always play an essential role. A more in depth study of the unavoidable coupling between "lateral" and "vertical" properties (or " $\ell-\Gamma$ interplay") may be a fruitful direction to follow for a better understanding of the physics of thin films and lamellar stacks. Some key assumptions in the field, namely

1. The various contributions to the disjoining pressure are additive (taken from 10

2. Surface coverage deduced from pressure-area isotherms is constant over the range of Newton black film thickness (as formulated in Ref. [11])

may have to be challenged for further progress.

\section{Acknowledgements}

Though being, administratively speaking, a chemist, I was given the opportunity to teach statistical physics in the physics department of the Bordeaux university. Taking shamelessly inspiration from Ref. [61], it is fair to say that 


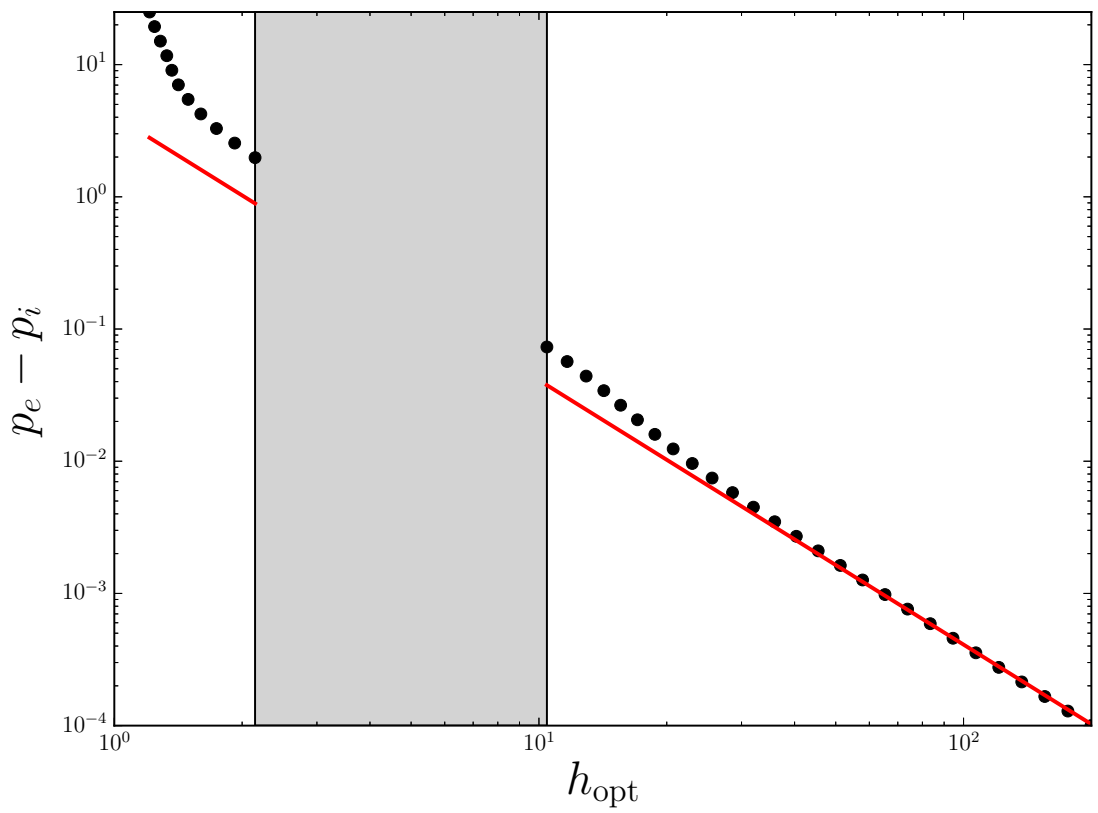

Figure 11: "Disjoining pressure"-black film core thickness equation of state in reduced units for the "toy" model, eq. (39). The red line corresponds to a power-law variation with exponent -2

Some crucial elements of this teaching experience germinated (part of) this contribution. The challenge of teaching engenders learning.

I am grateful to prof. Philippe TAMARAT and Brahim Lounis for this challenging opportunity.

\section{References}

[1] D. Langevin. Bubble coalescence in pure liquids and in surfactant solutions. Current Opinion in Colloid \& Interface Science, 20:92-97, 2015.

[2] B. Derjaguin and M. Kussakov. (in Russian). Bulletin de l'académie des sciences de l'URSS, série chimique, 5:1119, 1937.

[3] B. Derjaguin. On the repulsive forces between charged colloid particles and on the theory of slow coagulation and stability of lyophobe sols. Transactions of the Faraday Society, 35:203-215, 1940.

[4] A. Vrij. Possible mechanism for the spontaneous rupture of thin, free liquid films. Discussions of the Faraday Society, 42:23-33, 1966. 
[5] Charles Y. Young and Noel A Clark. Dynamics of freely suspended lyotropic films. I. An inelastic light scattering study of thermal surface fluctuations. Journal of Chemical Physics, 74:4171-4185, 1981.

[6] Vance Bergeron, Åsa Waltermo, and Per M. Claesson. Disjoining pressure measurements for foam films stabilized by a nonionic sugar-based surfactant. Langmuir, 12:1336-1342, 1996.

[7] John W. Cahn and John E. Hilliard. Free energy of a nonuniform system. i. interfacial free energy. Journal of Chemical Physics, 28:258-267, 1958.

[8] P.M. Chaikin and T.C. Lubensky. Principles of condensed matter physics. Cambridge University Press, 1995.

[9] O. Bélorgey and J. J. Benattar. Structural properties of soap black films investigated by x-ray reflectivity. Physical Review Letters, 66:313-316, 1991.

[10] Vance Bergeron. Forces and structure in thin liquid soap films. Journal of Physics: Condensed Matter, 11:R215, 1999.

[11] Seung Soon Jang and William A. Goddard III. Structures and properties of Newton black films characterized using molecular dynamics simulations. Journal of Physical Chemistry B, 110:7992-8001, 2006.

[12] A. Espert, R. v. Klitzing, P. Poulin, A. Colin, R. Zana, and D. Langevin. Behavior of soap films stabilized by a cationic dimeric surfactant. Langmuir, 14:4251-4260, 1998.

[13] D. Langevin, C. Marquez-Beltran, and J. Delacotte. Surface force measurements on freely suspended liquid films. Advances in Colloid and Interface Science, pages 124-134, 2011.

[14] Emmanuelle Rio and Anne-Laure Biance. Thermodynamic and mechanical timescales involved in foam film rupture and liquid foam coalescence. ChemPhysChem, 15:3692-3707, 2014.

[15] V. Luzzati, H. Mustacchi, A. Skoulios, and F. Husson. La structure des colloïdes d'association. I. les phases liquides-cristallines des systèmes amphiphile-eau. Acta Crystallographica, 13:660-667, 1960.

[16] D. M. Le Neveu, R. P. Rand, and V. A. Parsegian. Measurement of forces between lecithin bilayers. Nature, 259:601-603, 1976.

[17] J. S. Clunie, J. M. Corkill, and J. F. Goodman. Structure of black foam films. Discussions of the Faraday Society, 42:34-41, 1966.

[18] Philippe Poulin, Frédéric Nallet, Bernard Cabane, and Jérôme Bibette. Evidence for Newton black films between adhesive emulsion droplets. Physical Review Letters, 77:3248-3251, 1996. 
[19] J. Czarnecki, Kh. Khristov, J. Masliyah, N. Panchev, S.D. Taylor, and P. Tchoukova. Application of Scheludko-Exerowa thin liquid film technique to studies of petroleum $\mathrm{W} / \mathrm{O}$ emulsions. Colloids and Surfaces A: Physicochemical and engineering aspects, 519:2-10, 2017.

[20] R. Oda and J. D. Litster. Effect of symmetry on a three-component lamellar phase: X-ray scattering study. Journal de Physique II France, 7:815-824, 1997.

[21] O. Mondain-Monval, F. Leal-Calderon, and J. Bibette. Forces between emulsion droplets: Role of surface charges and excess surfactant. Journal de Physique II France, 6:1313-1329, 1996.

[22] G. Gotchev, T. Kolarov, Khr. Khristov, and D. Exerowa. Electrostatic and steric interactions in oil-in-water emulsion films from Pluronic surfactants. Advances in Colloid and Interface Science, 168:79 - 84, 2011.

[23] A. Ott, W. Urbach, D. Langevin, R. Ober, and M. Waks. Light scattering study of surfactant mutilayers elasticity. Role of incorporated proteins. Europhysics Letters, 12:395-400, 1990.

[24] Reinhard Strey, Reinhard Schomäcker, Didier Roux, Frédéric Nallet, and Ulf Olsson. Dilute lamellar and $\mathrm{L}_{3}$ phases in the binary water $\mathrm{C}_{12} \mathrm{E}_{5}$ system. Journal of the Chemical Society Faraday Transactions, 86:2253-2261, 1990.

[25] F. Reiss-Husson. Structure des phases liquide-cristallines de différents phospholipides, monoglycérides, sphingolipides, anhydres ou en présence d'eau. Journal of Molecular Biology, 25:363-382, 1967.

[26] V. A. Parsegian, N. Fuller, and R. P. Rand. Measured work of deformation and repulsion of lecithin bilayers. Proceeding of the National Academy of Sciences USA, 76:2750-2754, 1979.

[27] L. J. Lis, M. McAlister, N. Fuller, R. P. Rand, and V. A. Parsegian. Interactions between neutral phospholipid bilayer membranes. Biophysical Journal, 37:657-665, 1982.

[28] L. J. Lis, M. McAlister, N. Fuller, R. P. Rand, and V. A. Parsegian. Measurement of the lateral compressibility of several phospholipid bilayers. Biophysical Journal, 37:667-672, 1982.

[29] P. A. Kralchevsky, K. D. Danov, G. Broze, and A. Mehreteab. Thermodynamics of ionic surfactant adsorption with account for the counterion binding: Effect of salts of various valency. Langmuir, 15:2351-2365, 1999.

[30] R. Leite Rubim, B.B. Gerbelli, K. Bougis, C.L. Pinto de Oliveira, L. Navailles, F. Nallet, and E. Andreoli de Oliveira. Water activity in lamellar stacks of lipid bilayers: "Hydration forces" revisited. European Physical Journal E: Soft Matter, 39:3, 2016. 
[31] E. Leontidis, A. Aroti, L. Belloni, M. Dubois, and T. Zemb. Effects of monovalent anions of the Hofmeister series on DPPC lipid bilayers part II: Modeling the perpendicular and lateral equation-of-state. Biophysical Journal, 93:1591-1607, 2007.

[32] W. Helfrich. Steric interactions of fluid membranes in multilayer systems. Zeitschrift für Naturforschung, 33A:305-315, 1978.

[33] J. Clerk-Maxwell. Van der Waals on the continuity of the gaseous and liquid states. Nature, 10:477-480, 1874.

[34] S. T. Milner and D. Roux. Flory theory of the unbinding transition. Journal de Physique I France, 2:1741-1754, 1992.

[35] Ronald C. Chatelier and Allen P. Minton. Adsorption of globular proteins on locally planar surfaces: Models for the effect of excluded surface area and aggregation of adsorbed protein on adsorption equilibria. Biophysical Journal, 71:2367-2374, 1996.

[36] Robert D. Groot and Simeon D. Stoyanov. Equation of state of surfaceadsorbing colloids. Soft Matter, 6:1682-1692, 2010.

[37] Reinhard Lipowski and Stanislas Leibler. Unbinding transitions of interacting membranes. Physical Review Letters, 56:2541-2544, 1986.

[38] Rudi Podgornik and V. Adrian Parsegian. Thermal-mechanical fluctuations of fluid membranes in confined geometries: The case of soft confinement. Langmuir, 8:557-562, 1992.

[39] V. A. Parsegian and T. Zemb. Hydration forces: Observations, explanations, expectations, questions. Current Opinion in Colloid $\mathscr{E}$ Interface Science, 16:618-624, 2011.

[40] M. Dubois and T. Zemb. Phase behavior and scattering of double-chain surfactants in diluted aqueous solutions. Langmuir, 7:1352-1360, 1991.

[41] Th. Zemb, D. Gazeau, M. Dubois, and T. Gulik-Krzywicki. Critical behaviour of lyotropic liquid crystals. Europhysics Letters, 21:759, 1993.

[42] K. M. McGrath and C. J. Drummond. Polymerisation of liquid crystalline phases in binary surfactant/water systems. Colloid and Polymer Science, 274:316-333, 1996.

[43] Bruno F. B. Silva, Eduardo F. Marques, and Ulf Olsson. Lamellar miscibility gap in a binary catanionic surfactant-water system. Journal of Physical Chemistry B, 111:13520-13526, 2007.

[44] Bruno F. B. Silva, Eduardo F. Marques, Ulf Olsson, and Ramon Pons. Vesicle-to-micelle transition of salt-free catanionic amphiphiles. Langmuir, 26:3058-3066, 2010. 
[45] Frédéric Nallet, Didier Roux, and Jacques Prost. Hydrodynamics of lyotropic smectics: a dynamic light scattering study of dilute lamellar phases. Journal de Physique, 50:3147-3165, 1989.

[46] C. Ligoure, G. Bouglet, G. Porte, and O. Diat. Smectic compressibility of polymer-containing lyotropic lamellar phases: Experimental tool to study the thermodynamics of polymer confinement. Journal de Physique II France, 7:473-491, 1997.

[47] L. Porcar, C. Ligoure, and J. Marignan. Layer compression modulus of electrostatically stabilized lyotropic lamellar phases revisited: Application to the quantitative analysis of a polymer induced critical SmA-SmA phase separation. Journal de Physique II France, 7:493-501, 1997.

[48] M.-F. Ficheux, A.-M Bellocq, and F. Nallet. Elastic properties of polymerdoped dilute lamellar phases: A small-angle neutron scattering study. European Physical Journal E: Soft Matter, 4:315-326, 2001.

[49] Kévin Bougis. Fluctuations et interactions en situation de nano-confinement anisotrope. $\mathrm{PhD}$ thesis, université de Bordeaux - Universidade de São Paulo, 28 November 2016.

[50] C.J. Radke. Film and membrane-model thermodynamics of free thin liquid films. Journal of Colloid and Interface Science, 449:462-479, 2015.

[51] A. Prins, C. Arcuri, and M. van den Tempel. Elasticity of thin liquid films. Journal of Colloid and Interface Science, 24:84-90, 1967.

[52] V.B. Fainerman, R. Miller, and E.V. Aksenenko. Simple model for prediction of surface tension of mixed surfactant solutions. Advances in Colloid and Interface Science, 96:339-359, 2002.

[53] It will be convenient here to consider the air-water interface of a Langmuir trough ("thick limit") as splitted into two equivalent, half-area films.

[54] Karol J. Mysels and Malcolm N. Jones. Direct measurement of the variation of double-layer repulsion with distance. Discussions of the Faraday Society, 42:42-50, 1966.

[55] Dan Qu, Guillaume Brotons, Vera Bosio, Andreas Fery, Tim Salditt, Dominique Langevin, and Regine von Klitzing. Interactions across liquid thin films. Colloids and Surfaces A: Physicochemical and engineering aspects, 303:97-109, 2007.

[56] A. Sheludko. Thin liquid films. Advances in Colloid and Interface Science, 1:391-464, 1967.

[57] W.A.B Donners, J.B Rijnbout, and A Vrij. Calculations of van der Waals forces in thin liquid films using Lifshitz' theory. Journal of Colloid and Interface Science, 60:540 - 547, 1977. 
[58] Valeria Casteletto, Isabelle Cantat, Dipak Sarker, Richard Bausch, Daniel Bonn, and Jacques Meunier. Stability of soap films: Hysteresis and nucleation of black films. Physical Review Letters, 90:048302-1-048302-4, 2003.

[59] Judith Schlarmann, Cosima Stubenrauch, and Reinhard Strey. Correlation between film properties and the purity of surfactants. Physical Chemistry Chemical Physics, 5:184-191, 2003.

[60] An aspect ratio parameter equal to $50 \mathrm{~nm}$ is close to $\ell=60$ in reduced units and is reached when the differential pressure exceeds $\approx 1.3 \times 10^{-3}$-or about $10 \mathrm{kPa}$ for the values chosen when drawing Fig. 9

[61] C.J. Radke. Gibbs adsorption equation for planar fluid-fluid interfaces: Invariant formalism. Advances in Colloid and Interface Science, 222:600614, 2015. 